\title{
Systems approaches to uncovering in vivo state of the TB bacillus
}

\author{
Johnjoe McFadden \\ From Immunodiagnosis of Tuberculosis: New Questions, New Tools \\ Virginia, VA, USA. 21-23 September 2008
}

Research related to system approaches to uncovering the characteristics of the TB bacillus grown in vivo could ultimately be useful in discovering new immunodiagnostic approaches. There are important differences between a classic molecular biology view of genes and phenotypes (one gene/one enzyme) and the systems-level vision which focuses on identifying all the interactions between genes and phenotypes. The systems approach requires an in silico model to identify all the possible genes and phenotypes. We have constructed such a model and are using it to investigate differences between in vivo Mycobacterium tuberculosis (M.tb) and in vitro M.tb. It is known that in vitro M.tb behaves differently from in vivo M.tb. For instance, M.tb in vitro is relatively easy to rapidly kill with a single drug. Not in vivo, however: the latter gives a two-hit pattern, suggesting that a subpopulation of the bacteria is harder to kill, even though they are still genetically susceptible. Is this due to different gene expression patterns? If so, how do we identify these? The genes that are specific to in vivo TB may be useful in distinguishing between infection and exposure and may be promising targets for new drugs or imunodiagnostics. Using a systems biology approach, our team has developed an approach that could be used to identify these antigens.

We found some aspects of the in vivo state could be simulated in vitro, using a culture system called a chemostat with a glycerol substrate to control the growth rate of the bacillus. At a slow rate, the bacillus developed phenotypic drug tolerance. We then developed an in silico genome-scale model, basically a mathematical model of linked metabolic pathways to predict how genes interact to generate the metabolism of the cell.

Correspondence: J.Mcfadden@surrey.ac.uk

Faculty of Health and Medical Sciences, University of Surrey, Guildford,

Surrey, GU2 7XH, UK
The model was tested by comparing gene essentiality predictions with experimental data where it was shown that the in silico model generated $78 \%$ correct predictions. It was also able to predict the existence of a novel metabolic pathway operating in slow-growing M.tb. The M.tb model can also be used to interrogate transcriptome date to identify signal metabolites. Our research indicates that a systems approach may prove useful in identifying novel immunodiagnostics for determining whether a patient is infected with active or latent TB. However, development of such immunodiagnostics will first require identification of differences between M.tb growing in acute and chronic lesions. Systems-based approaches, such as we have described here, could be used to identify such differences.

Published: 17 December 2010

doi:10.1186/1753-6561-4-S3-O5

Cite this article as: McFadden: Systems approaches to uncovering in vivo state of the TB bacillus. BMC Proceedings 2010 4(Suppl 3):O5.

Submit your next manuscript to BioMed Central and take full advantage of:

- Convenient online submission

- Thorough peer review

- No space constraints or color figure charges

- Immediate publication on acceptance

- Inclusion in PubMed, CAS, Scopus and Google Scholar

- Research which is freely available for redistribution 